\title{
N91-21078
}

\section{DISTRIBUTED MINIMAL RESIDUAL (DMR) METHOD FOR ACCELERATION OF ITERATIVE ALGORITHMS}

Seungsoo Lee and George S. Dulikravich

Pennsylvania State University

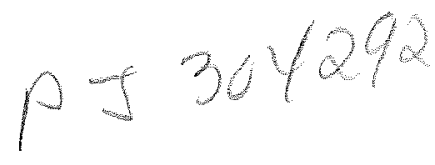

University Park, Pennsylvania, U.S.A.

\begin{abstract}
A new method for enhancing convergence rate of iterative algorithms for the numerical integration of systems of partial differential equations has been developed. It is termed the Distributed Minimal Residual (DMR) method and it is based on general Krylov subspace methods. The DMR method differs from the Krylov subspace methods by the fact that the iterative acceleration factors are different from equation to equation in the system. At the same time, the DMR method can be viewed as an incomplete Newton iteration method. The DMR method has been applied to Euler equations of gasdynamics and incompressible Navier-Stokes equations. All numerical test cases were obtained using either explicit four stage Runge-Kutta or Euler implicit time integration. The formulation for the DMR method is general in nature and can be applied to explicit and implicit iterative algorithms for arbitrary systems of partial differential equations.
\end{abstract}

\section{INTRODUCTION}

After linearization caused by the discretization, the systems of governing equations associated with , say, fluid flows are recast into the following linear system of algebraic equations

$$
\mathbf{A X}=\mathbf{b}
$$

where $X$ is the vector of unknowns and $A$ is an $N \times N$ matrix which depends on the discretized scheme, and is assumed to be non-singluar. The matrix $\mathbf{A}$ is usually sparse and as $\mathbf{N}$ becomes larger, it is not economical to solve the system of equations directly. Instead, iterative methods are usually utilized.

The Conjugate Gradient (CG) method and the Conjugate Residual (CR) method, are widely used for approximating the solution of the system (Huynh, ref. 1; Faddeev and Faddeeva, ref. 2). Both methods give the exact solution in at most $\mathrm{N}$ steps in the absence of round-off errors. However, the 
$\mathrm{CG}$ method and the $\mathrm{CR}$ method require the matrix $\mathbf{A}$ to be symmetric, positive definite. A large number of generalizations of these methods applicable to systems with a non-symmetric matrix have been made. The success of the generalization of the CG and CR methods is reflected in the introduction of a series of algorithms capable of treating non-symmetric problems (OTHMIN by Vinsome, ref. 3; ORTHDIR and ORTHRES by Young and Jea, ref. 4; GMRES by Saad and Schultz, ref. 5; Wigton et al., ref. 6). The Minimal Residual method (Hafez, ref. 7) and the Generalized Nonlinear Minimal Residual method (Huang and Dulikravich, ref. 8) can be thought of as generalizations of the conjugate residual method.

In this paper, a new method of enhancing convergence rate of iterative algorithms for systems of partial differential equations is developed. The method is entitled Distributed Minimal Residual (DMR) method (Lee et al., ref. 9-14) and it is related to a general Krylov subspace method from which it differs in two aspects. First, the DMR method attempts to improve on a straight application of a Krylov subspace method by using a separate sequence of acceleration factors for each equation in the system. In application of the DMR method to Euler equations of inviscid gasdynamics, for example, the acceleration factors for continuity equation differ from those for two momentum equations and for energy equation. This approach requires fewer consecutive solutions to be stored. Effectively, the DMR method periodically preconditions the system. Second, the DMR method does not involve the orthogonalization procedure which most of Krylov subspace methods utilize to reduce the number of numerical operations. The DMR method uses corrections from only two or three consecutive solutions for a successful application.

The prime objective of this paper is to develop the theory of the DMR method and to examine the effectiveness of the DMR method by applying it to different systems of partial differential equations: Euler equations of inviscid gasdynamics and incompressible flow Navier-Stokes equations. RungeKutta time stepping method and Euler implicit method were used as two basic iterative algorithms.

\section{DISTRIBUTED MINIMAL RESIDUAL (DMR) METHOD}

Let us consider a system of partial differential equations that are integrated iteratively so that their residual vector at iteration level $t$ is given by

$$
\mathbf{R}^{\mathrm{t}}=\frac{\partial \mathbf{E}^{\mathrm{t}}}{\partial \mathrm{x}}+\frac{\partial \mathbf{F}^{\mathrm{t}}}{\partial \mathrm{y}}+\frac{\partial \mathbf{G}^{\mathrm{t}}}{\partial \mathrm{z}}
$$

where $\mathbf{E}^{\mathrm{t}}, \mathrm{F}^{\mathrm{t}}, \mathrm{G}^{\mathrm{t}}$ are the generalized flux vectors (at iteration level $\mathrm{t}$ ) that act in the directions $\mathrm{x}, \mathrm{y}, \mathrm{z}$, respectively. The future residual at iteration level $t+1$ is given by 


$$
\mathbb{R}^{\mathrm{t}+1}={\frac{\partial \mathbb{E}^{\mathrm{t}+1}}{\partial \mathrm{x}}}+\frac{\partial \mathbb{F}^{\mathrm{t}+1}}{\partial \mathrm{y}}+\frac{\partial \mathbf{G}^{\mathrm{t}+1}}{\partial \mathrm{z}}
$$

Assume that each component of the solution vector at iteration level $t+1$ is extrapolated from the corresponding previous $\mathrm{M}$ consecutive iteration levels. Then, we can say that

$$
\begin{aligned}
\mathrm{q}_{1}^{\mathrm{t}+1}=\mathrm{q}_{1}^{\mathrm{t}}+\omega_{1}^{1} \Delta \mathrm{q}_{1}^{1}+\omega_{1}^{2} \Delta \mathrm{q}_{1}^{2}+\cdots+\omega_{1}^{\mathrm{M}} \Delta \mathrm{q}_{1}^{\mathrm{M}} \\
\mathrm{q}_{2}^{\mathrm{t}+1=}=\mathrm{q}_{2}^{\mathrm{t}}+\omega_{2}^{1} \Delta \mathrm{q}_{2}^{1}+\omega_{2}^{2} \Delta \mathrm{q}_{2}^{2}+\cdots+\omega_{2}^{\mathrm{M}} \Delta \mathrm{q}_{2}^{\mathrm{M}} \\
\quad \cdots \cdots \cdots \cdots \cdots \cdots \cdots \cdots \cdots \cdots \cdots \\
\mathrm{q}_{\mathrm{L}}^{\mathrm{t}+1}=\mathrm{q}_{\mathrm{L}}^{\mathrm{t}}+\omega_{\mathrm{L}}^{1} \Delta \mathrm{q}_{\mathrm{L}}^{1}+\omega_{\mathrm{L}}^{2} \Delta \mathrm{q}_{\mathrm{L}}^{2}+\cdots+\omega_{\mathrm{L}}^{\mathrm{M}} \Delta \mathrm{q}_{\mathrm{L}}^{\mathrm{M}}
\end{aligned}
$$

Here, the subscripts $1,2,3, \ldots, \mathrm{L}$ designate the particular component of the solution vector $\mathbf{Q}$, that is, the particular equation in the system. The superscripts $1,2,3, \ldots, \mathrm{M}$ designate the particular iteration level counting backward from the present iteration level, $t$. Thus, the superscript 1 means the first previous iteration level. The superscript 2 means the second previous iteration level, etc. This can be expressed in a more compact form as

$$
\mathbf{Q}^{\mathrm{t}+1}=\mathbf{Q}^{\mathrm{t}}+\sum_{\mathrm{m}=1}^{\mathrm{M}} \Theta^{\mathrm{m}}
$$

where

$$
\Theta^{\mathrm{n}}=\left[\begin{array}{c}
\omega_{1}^{\mathrm{n}} \Delta_{1}^{\mathrm{n}} \\
\omega_{2}^{\mathrm{n}} \Delta_{2}^{\mathrm{n}} \\
\cdot \\
\cdot \\
\omega_{\mathrm{L}}^{\mathrm{n}} \Delta_{\mathrm{L}}^{\mathrm{n}}
\end{array}\right]=\omega_{1}^{\mathrm{n}}\left[\begin{array}{c}
\Delta_{1}^{\mathrm{n}} \\
0 \\
\cdot \\
\cdot \\
0
\end{array}\right]+\omega_{2}^{\mathrm{n}}\left[\begin{array}{c}
0 \\
\Delta_{2}^{\mathrm{n}} \\
\cdot \\
\cdot \\
0
\end{array}\right]+\cdots+\omega_{1}^{\mathrm{n}}\left[\begin{array}{c}
0 \\
0 \\
\cdot \\
\cdot \\
\Delta_{\mathrm{L}}^{\mathrm{n}}
\end{array}\right]
$$

Here, $\omega$ 's are the acceleration (weighting) factors to be calculated, $\Delta$ 's are the iterative corrections computed with the original non-accelerated scheme, $\mathrm{M}$ denotes the total number of consecutive time steps from which the corrections are combined. 
Using Taylor series expansion in time for $\mathbb{R}^{\mathfrak{t}+1}$ and truncating the terms that are higher than second order in $\Delta t$, Eq. 3 becomes approximately

$$
\mathbf{R}^{\mathrm{t}+1}=\mathbf{R}^{\mathrm{t}}+\sum_{\mathrm{m}=1}^{\mathrm{M}}\left[\frac{\partial}{\partial \mathrm{x}} \mathbf{A}^{\mathrm{t}} \cdot+\frac{\partial}{\partial y} \mathbf{B}^{\mathrm{t}} \cdot+\frac{\partial}{\partial z} \mathbf{C}^{\mathrm{t}} \cdot\right] \Theta^{\mathrm{m}}
$$

The global domain residual can be defined as

$$
\mathbf{R}^{\mathrm{t}}=\sum_{\mathrm{D}} \mathbf{R}^{\mathrm{t}^{\mathrm{T}}} \mathbf{R}^{\mathrm{t}}
$$

where $\sum_{D}$ denotes summation over the computational domain $D$, and the superscript $T$ represents transpose of a vector. In order to minimize the future global residual, $\mathrm{R}^{\mathrm{t}+1}$, the $\omega^{\prime}$ s are determined from the following conditions

$$
\frac{\partial R^{t+1}}{\partial \omega_{r}^{m}}=0
$$

From Eq. 8 this leads to

$$
\begin{gathered}
-\sum_{D} \mathbf{R}^{\mathrm{t}^{\mathrm{T}}}\left[\frac{\partial}{\partial \mathrm{x}} \mathbf{A}^{\mathrm{t}} \cdot+\frac{\partial}{\partial y} \mathbf{B}^{\mathrm{t}} \cdot+\frac{\partial}{\partial z} \mathbf{C}^{\mathrm{t}} \cdot\right] \frac{\partial \Theta^{\mathrm{m}}}{\partial \omega_{r}^{\mathrm{m}}} \\
=\sum_{D} \sum_{\mathrm{n}}^{\mathrm{M}}\left\{\left[\frac{\partial}{\partial x} \mathbf{A}^{\mathrm{t}} \cdot+\frac{\partial}{\partial y} \mathbf{B}^{\mathrm{t}} \cdot+\frac{\partial}{\partial z} \mathbf{C}^{\mathrm{t}} \cdot\right] \Theta^{\mathrm{n}}\right\}^{\mathrm{T}} \cdot \\
\left\{\left[\frac{\partial}{\partial \mathrm{x}} \mathbf{A}^{\mathrm{t}} \cdot+\frac{\partial}{\partial y} \mathbf{B}^{\mathrm{t}} \cdot+\frac{\partial}{\partial z} \mathbf{C}^{\mathrm{t}} \cdot\right] \frac{\partial \Theta^{\mathrm{m}}}{\partial \omega_{r}^{\mathrm{m}}}\right\}
\end{gathered}
$$

where

$$
\frac{\partial \Theta^{\mathrm{m}}}{\partial \omega_{\mathrm{r}}^{\mathrm{m}}}=\left[\Delta_{\mathrm{p}}^{\mathrm{m}} \delta_{\mathrm{pr}}\right]
$$

and $\delta_{\mathrm{pr}}$ is the Kronecker delta. However, from Eq. 6 we have that 


$$
\Theta^{n}=\sum_{q}^{L} \omega_{q}^{n} \frac{\partial \Theta^{n}}{\partial \omega_{q}^{n}}
$$

Noticing that $\frac{\partial \Theta^{n}}{\partial \omega_{q}^{n}}$ is not a function of $\omega$, it follows that

$$
\begin{gathered}
-\sum_{D} \mathbf{R}^{\mathrm{t}^{\mathrm{T}}}\left[\frac{\partial}{\partial \mathrm{x}} \mathbf{A}^{\mathrm{t}} \cdot+\frac{\partial}{\partial y} \mathbf{B}^{\mathrm{t}} \cdot+\frac{\partial}{\partial z} \mathbf{C}^{\mathrm{t}} \cdot\right] \frac{\partial \Theta^{\mathrm{m}}}{\partial \omega_{r}^{\mathrm{m}}} \\
=\sum_{D} \sum_{\mathrm{n}}^{\mathrm{M}} \sum_{\mathrm{q}}^{\mathrm{L}} \omega_{\mathrm{q}}^{\mathrm{n}}\left\{\left[\frac{\partial}{\partial \mathrm{x}} \mathbf{A}^{\mathrm{t}} \cdot+\frac{\partial}{\partial y} \mathbf{B}^{\mathrm{t}} \cdot+\frac{\partial}{\partial z} \mathbf{C}^{\mathrm{t}} \cdot \frac{\partial \Theta^{\mathrm{n}}}{\partial \omega_{\mathrm{q}}^{\mathrm{n}}}\right\}^{\mathrm{T}}\right. \\
\left\{\left[\frac{\partial}{\partial \mathrm{x}} \mathbf{A}^{\mathrm{t}} \cdot+\frac{\partial}{\partial y} \mathbf{B}^{\mathrm{t}} \cdot+\frac{\partial}{\partial z} \mathbf{C}^{\mathrm{t}} \cdot\right]_{\left.\frac{\partial \Theta^{\mathrm{m}}}{\partial \omega_{r}^{\mathrm{m}}}\right\}}\right.
\end{gathered}
$$

Let

$$
\mathbf{a}_{\mathrm{q}}^{\mathrm{n}}=\left[\frac{\partial}{\partial \mathrm{x}} \mathbf{A}^{\mathrm{t}} \cdot+\frac{\partial}{\partial y} \mathbf{B}^{\mathrm{t}} \cdot+\frac{\partial}{\partial z} \mathbf{C}^{\mathrm{t}} \cdot\right] \frac{\partial \Theta^{\mathrm{n}}}{\partial \omega_{\mathrm{q}}^{\mathrm{n}}}
$$

Then Eq. 13 becomes

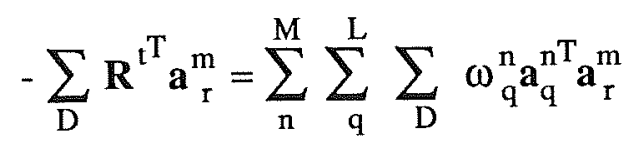

For simplicity, let

and

$$
\begin{aligned}
& c_{q r}^{n m}=\sum_{D} a_{q}^{n_{q}^{T}} a_{r}^{m} \\
& b_{r}^{m}=-\sum_{D} R^{t^{1 T}} \mathbf{a}_{r}^{m}
\end{aligned}
$$

Then, the system of algebraic equations (Eq. 15) can be written as

$$
\sum_{n}^{M} \sum_{q}^{L} \omega_{q}^{n} c_{q r}^{n m}=b_{r}^{m}
$$




$$
\left[\begin{array}{ccccccc}
c_{11}^{11} & c_{21}^{11} & \ldots & c_{\mathrm{L} 1}^{11} & c_{11}^{21} & \ldots & c_{\mathrm{L} 1}^{\mathrm{M} 1} \\
c_{12}^{11} & c_{22}^{11} & \ldots & c_{\mathrm{L} 2}^{11} & c_{12}^{21} & \ldots & c_{\mathrm{L} 2}^{\mathrm{M} 1} \\
\vdots & \vdots & \vdots & \vdots & \vdots & \vdots & \vdots \\
c_{1 \mathrm{~L}}^{11} & c_{2 \mathrm{~L}}^{11} & \ldots & c_{\mathrm{LL}}^{11} & c_{1 \mathrm{~L}}^{21} & \ldots & c_{\mathrm{LL}}^{\mathrm{M} 1} \\
c_{11}^{12} & c_{21}^{12} & \ldots & c_{\mathrm{L} 1}^{12} & c_{11}^{22} & \ldots & c_{\mathrm{Ll}}^{11} \\
\vdots & \vdots & \vdots & \vdots & \vdots & \vdots & \vdots \\
c_{1 \mathrm{~L}}^{1 \mathrm{M}} & c_{2 \mathrm{~L}}^{1 \mathrm{M}} & \ldots & c_{\mathrm{LL}}^{1 \mathrm{M}} & c_{1 \mathrm{~L}}^{11} & \ldots & c_{\mathrm{LL}}^{\mathrm{MM}}
\end{array}\right]\left[\begin{array}{c}
\omega_{1}^{1} \\
\omega_{2}^{1} \\
\vdots \\
\omega_{\mathrm{L}}^{1} \\
\omega_{1}^{2} \\
\vdots \\
\omega_{\mathrm{L}}^{\mathrm{M}}
\end{array}\right]=\left[\begin{array}{c}
b_{1}^{1} \\
b_{2}^{1} \\
\vdots \\
b_{\mathrm{L}}^{1} \\
b_{1}^{2} \\
\vdots \\
b_{\mathrm{L}}^{\mathrm{M}}
\end{array}\right]
$$

representing the system of $L \times M$ linear algebraic equations for the $L \times M$ optimum acceleration factors $\omega$. For example, if we are periodically to combine corrections from $\mathrm{M}=2$ consecutive time steps to extrapolate the solution and to solve a system of $\mathrm{L}=4$ partial differential equations, we need to solve simultaneously $L \times M=8$ algebraic equations for 8 values of $\omega$.

Notice that when the convergence is achieved, the b's become zero (Eq. 17), thus making the $\omega$ 's zero. In other words, the accuracy of the fully converged solution will not be affected by using the DMR method. Furthermore, if the matrix $c_{q_{r}}^{\mathrm{nm}}$ is positive definite, it can be shown easily that the $\omega$ 's minimize the global residual, $\mathrm{R}^{\mathrm{t}+1}$, at iteration level $\mathrm{t}+1$. Using a different sequence of acceleration factors for each partial differential equation in the original system is equivalent to using a different time step for each equation or selectively preconditioning the system. The DMR method, therefore, can be understood as the combination of a preconditioning method and a Krylov subspace method. Also, we can think of the DMR method as an incomplete Newton iteration. This point can be illustrated by the following fact. When the acceleration factors vary not only from equation to equation, but also from grid point to grid point, and when we use just one solution in the DMR formulation, it can be shown that the DMR method is equivalent to the Newton iterations.

\section{APPLICATION OF THE DMR METHOD TO EULER EQUATIONS OF GASDYNAMICS}

The introduction of the successful numerical algorithms such as the Euler implicit method and the explicit Runge-Kutta time stepping method made it relatively inexpensive to perform the numerical 
integration of the systems of partial differential equations governing compressible flows. Most of such algorithms, however, suffer from slow convergence at low Mach numbers. The reasons for this are the rapidly increased stiffness and the singular behavior of the original system of compressible flow equations at low Mach numbers. The singular behavior of the system near Mach number zero can be removed by eliminating the singularity of the system by a perturbation technique (Briley, ref. 15; Choi, ref. 16). The stiffness of the system at low Mach numbers can be reduced by preconditioning the system (Turkel, ref. 17; Choi, ref. 16). The DMR method is used to alleviate the difficulty associated with the increased stiffness of the Euler equations for low Mach number compressible flows.

\section{Euler Equations for Compressible flows}

The Euler equations for a two-dimensional unsteady inviscid flow expressed in a generalized nonorthogonal curvilinear coordinates $(\xi, \eta)$ without body forces or heat transfer, can be written in a vector form as

$$
\frac{\partial \widetilde{\mathbf{Q}}}{\partial \mathrm{t}}+\frac{\partial \widetilde{\mathbf{E}}}{\partial \xi}+\frac{\partial \widetilde{\mathbf{F}}}{\partial \eta}=0
$$

where

$$
\widetilde{\mathbf{Q}}=\frac{1}{\mathrm{~J}}\left[\begin{array}{c}
\rho \\
\rho \mathrm{u} \\
\rho \mathrm{v} \\
\mathrm{e}
\end{array}\right] \quad \widetilde{\mathbf{E}}=\frac{1}{\mathrm{~J}}\left[\begin{array}{c}
\rho U \\
\rho U \mathrm{u}+\mathrm{p} \xi_{\mathrm{x}} \\
\rho U \mathrm{U}+\mathrm{p} \xi_{\mathrm{y}} \\
(\mathrm{e}+\mathrm{p}) \mathrm{U}
\end{array}\right] \quad \widetilde{\mathbf{F}}=\frac{1}{\mathrm{~J}}\left[\begin{array}{c}
\rho V \\
\rho V u+p \eta_{\mathrm{x}} \\
\rho V v+p \eta_{y} \\
(\mathrm{e}+\mathrm{p}) \mathrm{V}
\end{array}\right]
$$

The subscripts $x$ and $y$ represent first (partial) derivatives with respect to $x$ and $y$, respectively. Here, $p$ is the density, $\mathrm{p}$ is the thermodynamic pressure, $\mathrm{e}$ is the total energy per unit volume, while $\mathrm{u}$, and $\mathrm{v}$ are the Cartesian velocity components along $\mathrm{x}$ and $\mathrm{y}$ axis, respectively. $\mathrm{J}$ is the Jacobian determinant, $\frac{\partial(\xi, \eta)}{\partial(x, y)}$, while $U$ and $V$ are the contravariant velocity vector components defined as

$$
\left[\begin{array}{l}
\mathrm{U} \\
\mathrm{V}
\end{array}\right]=\left[\begin{array}{cc}
\xi_{\mathrm{x}} & \xi_{\mathrm{y}} \\
\eta_{\mathrm{x}} & \eta_{\mathrm{y}}
\end{array}\right]\left[\begin{array}{l}
\mathrm{u} \\
\mathrm{v}
\end{array}\right]
$$




\section{Numerical Algorithm}

The artificial dissipation suggested by Steger and Kutler (ref. 18) was used in the form

$$
\mathrm{D}(\mathrm{J} \tilde{\mathbf{Q}})=\frac{\varepsilon}{8 \mathrm{~J} \Delta \mathrm{t}} \nabla^{4}[\mathrm{~J} \tilde{\mathbf{Q}}]
$$

where $\nabla^{4}$ is the biharmonic differential operator in $\xi, \eta$ coordinates and $\varepsilon$ is a parameter. The residual vector $\hat{\mathbf{R}}$ of Euler equations for compressible flow including the artificial dissipation is

$$
\hat{\mathbf{R}}=\frac{\partial \widetilde{\mathbf{E}}}{\partial \xi}+\frac{\partial \widetilde{\mathbf{F}}}{\partial \eta}+\frac{\varepsilon}{8 \mathrm{~J} \Delta \mathrm{t}} \nabla^{4}[\mathrm{~J} \widetilde{\mathbf{Q}}]
$$

After discretization, the governing equations become a set of ordinary differential equations, which can be integrated by the Runge-Kutta time stepping method (Jameson et al., ref. 18).

$$
\begin{gathered}
\widetilde{\mathbf{Q}}^{0}=\widetilde{\mathbf{Q}}^{\mathrm{t}} \\
\Delta \widetilde{\mathbf{Q}}^{\mathrm{k}}=-\alpha_{\mathbf{k}} \Delta \mathrm{t} \hat{\mathbf{R}}^{\mathrm{k}-1} \quad \mathrm{k}=1,2, \ldots, \mathbf{K} \\
\widetilde{\mathbf{Q}}^{\mathrm{t}+1}=\widetilde{\mathbf{Q}}^{\mathrm{t}}+\Delta \widetilde{\mathbf{Q}}^{\mathrm{K}}
\end{gathered}
$$

where $\alpha_{\mathrm{k}}$ are the coefficients for each of the K stages of the Runge-Kutta scheme required to advance the solution from the time level $t$ to the time level $t+1$. For example, $\alpha_{k}=1 / 4,1 / 3,1 / 2$ and 1 for the four stage Runge-Kutta scheme.

The time steps for each direction are estimated (MacCormack and Baldwin, ref. 19) from

$$
\Delta t_{\xi}=\frac{C F L}{|U|+c\left(\xi_{x}^{2}+\xi_{y}^{2}\right)^{1 / 2}} \quad \Delta t_{\eta}=\frac{C F L}{|V|+c\left(\eta_{x}^{2}+\eta_{y}^{2}\right)^{1 / 2}}
$$

where $\mathrm{c}$ is the local speed of sound and CFL is the Courant-Friedrichs-Lewy number. The maximum time step is given as

$$
\Delta \mathrm{t}=\frac{\Delta \mathrm{t}_{\xi} \Delta \mathrm{t}_{\eta}}{\Delta \mathrm{t}_{\xi}+\Delta \mathrm{t}_{\eta}}
$$


The implicit characteristic boundary procedure of Chakravarthy (ref. 20) was used, though the scheme itself is explicit. Entropy per unit mass $\left(s=p / \rho^{\gamma}\right)$, total enthalpy per unit mass, $h=(e+p) / \rho$, and flow angle $(\tan (\alpha)=v / u)$ are specified at the inflow boundary. For a subsonic downstream outflow boundary $\left(\xi=\right.$ constant), the equation corresponding to the negative eigenvalue, $U-c\left(\xi_{x}^{2}+\xi_{y}^{2}\right)^{1 / 2}$, is substituted with a constant back pressure, $\mathrm{p}_{\mathrm{b}}$. For a solid wall boundary $(\eta=$ constant $)$, the equation corresponding to the positive eigenvalue, $\mathrm{V}+\mathrm{c}\left(\eta_{\mathrm{x}}^{2}+\eta_{\mathrm{y}}^{2}\right)^{1 / 2}$, is substituted with a tangency boundary condition, $\mathrm{V}=0$.

Upon applying the DMR method to the system of Euler equation of gasdynamics, Eq. 14, becomes

$$
\mathbf{a}_{\mathrm{q}}^{\mathrm{m}}=\left[\frac{\partial}{\partial \xi} \tilde{\mathbf{A}}^{\mathrm{t}} \cdot+\frac{\partial}{\partial \eta} \widetilde{\mathbf{B}}^{\mathrm{t}} \cdot+\mathrm{DJ} \cdot\right] \frac{\partial \Theta^{\mathrm{m}}}{\partial \omega_{\mathrm{q}}^{\mathrm{m}}}
$$

where $\widetilde{\mathbf{A}}$ and $\widetilde{\mathbf{B}}$ are the Jacobian matrices in the transformed coordinates

$$
\widetilde{\mathbf{A}}=\frac{\partial \widetilde{\mathbf{E}}}{\partial \widetilde{\mathbf{Q}}} \quad \widetilde{\mathbf{B}}=\frac{\partial \widetilde{\mathbf{F}}}{\partial \widetilde{\mathbf{Q}}}
$$

\section{Results for Compressible Euler Equations}

A two-dimensional flow analysis code has been developed in FORTRAN according to the previous theory for Euler equations of gasdynamics using finite differencing. All computational results were obtained on CRAY-YMP at NAS facility using automatic vectorization.

The test case for the code was flow around a circular cylinder. The outer boundary of the computational domain was located at 20 times the radius of the cylinder. A $66 \times 32$ cell computational grid was used in this test case. The computations were performed with and without the DMR method in conjunction with the four stage Runge-Kutta (RK) scheme. The convergence histories are plotted in terms of the number of iterations and in terms of the CPU time (Fig. 1). The maximum allowable CFL number $(\mathrm{CFL}=2.8$ ) was used in both accelerated and non-accelerated computations. The free stream Mach number was chosen to be 0.05 which is practically an incompressible flow. The DMR method saves over $60 \%$ of total CPU time in this critical flow test case. The surface pressure coefficient (Fig. 2) matches well with the incompressible potential flow solution. 

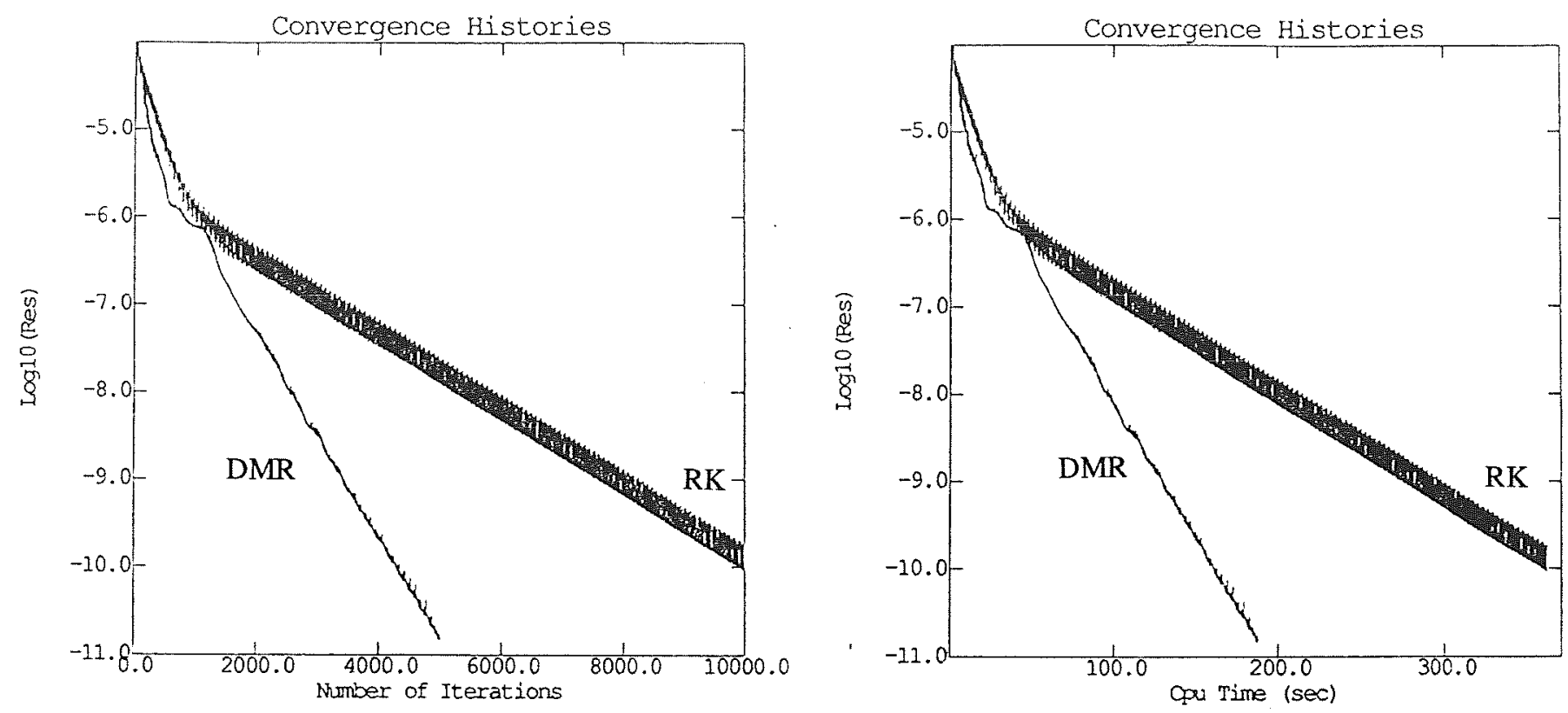

Figure 1 Convergence histories for the inviscid flow around a circular cylinder with $\mathrm{M}_{\infty}=0.05$

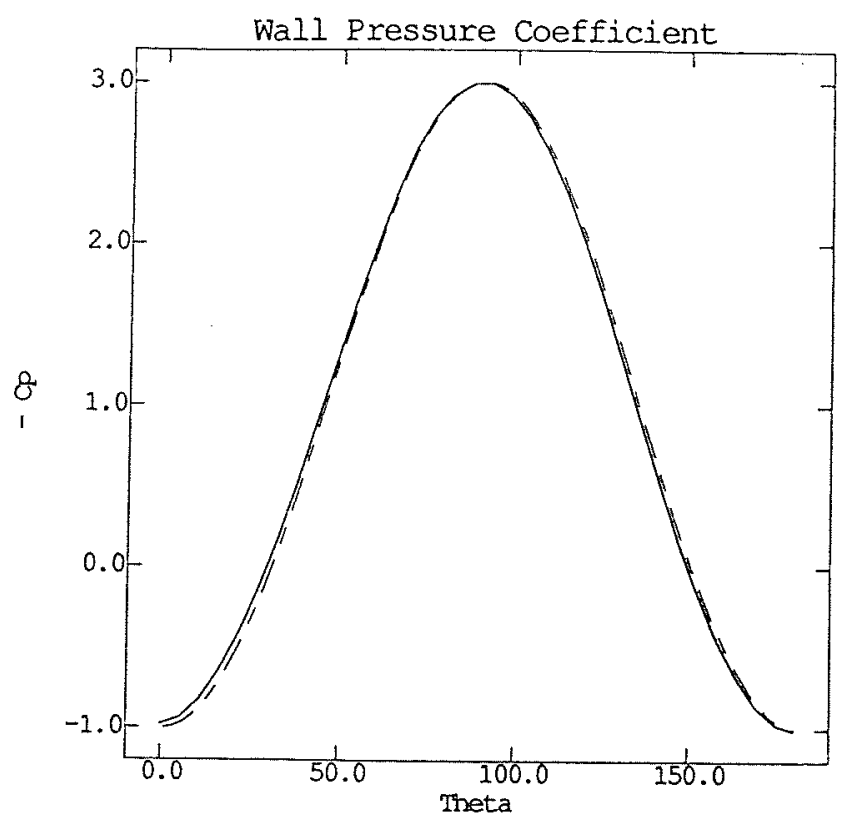

Figure 2 Wall pressure coefficient distribution for the inviscid flow around a circular cylinder: numerical with $\mathrm{M}_{\infty}=0.05$ (solid line); analytical with $\mathrm{M}_{\infty}=0$ (dotted line)

\section{APPLICATION OF THE DMR METHOD} TO INCOMPRESSIBLE NAVIER-STOKES EQUATIONS

The main difficulty associated with the incompressible flow computations is caused by the absence of a time derivative term in the continuity equation. One of the methods for solving the incompressible 
Navier-Stokes equations was originated by Chorin (ref. 21). In this concept, an artificially time dependent derivative term $\frac{\partial(p / \beta)}{\partial t}$ is added to the continuity equation with a user specified control parameter $\beta$. The artificial time derivative diminishes as the solution converges to its steady state. The added term forces the system to be of a mixed parabolic-hyperbolic type, which allows the use of time marching techniques. Later, Choi and Merkle (ref. 22), and Kwak et al. (ref. 23) used an Alternating Direction Implicit (ADI) method in conjunction with the artificial compressibility method.

\section{Incompressible Flow Navier-Stokes Equations}

The two-dimensional Navier-Stokes equations in a general non-orthogonal curvilinear coordinates $\xi, \eta$ are given as

$$
\frac{\partial \widetilde{\mathbf{Q}}}{\partial t}+\frac{\partial \widetilde{\mathbf{E}}}{\partial \xi}+\frac{\partial \widetilde{\mathbf{F}}}{\partial \eta}=D^{2}(\mathbf{J} \widetilde{\mathbf{Q}})
$$

The solution vector and the flux vectors in the transformed coordinates are given as

$$
\widetilde{\mathbf{Q}}=\frac{1}{J}\left[\begin{array}{c}
\mathrm{J} / \beta \\
\mathrm{u} \\
\mathrm{v}
\end{array}\right] \quad \widetilde{\mathbf{E}}=\frac{1}{\mathrm{~J}}\left[\begin{array}{c}
\mathrm{U} \\
\mathrm{Uu}+\xi_{\mathrm{x}} \mathrm{p} \\
\mathrm{Uv}+\xi_{\mathrm{y}} \mathrm{p}
\end{array}\right] \quad \widetilde{\mathbf{F}}=\frac{1}{\mathrm{~J}}\left[\begin{array}{c}
\mathrm{V} \\
\mathrm{Vu}+\eta_{\mathrm{x}} \mathrm{p} \\
\mathrm{Vv}+\eta_{\mathrm{y}} \mathrm{p}
\end{array}\right]
$$

where $\mathrm{p}$ is the pressure. Notice that the artificial compressibility has been added in the continuity equation. The physical viscous terms in the general coordinates are given by

$$
D^{2}(\mathrm{~J} \widetilde{\mathbf{Q}})=\left[\frac{\mathrm{S}}{\mathrm{J}} \mathrm{g}_{\mathrm{ij}}(\mathrm{J} \widetilde{\mathbf{Q}})_{, \mathrm{j}}\right]_{, \mathrm{i}}
$$

where $g_{i j}$ is the contravariant matrix tensor

$$
\mathrm{g}_{\mathrm{ij}}=\nabla \mathrm{x}_{\mathrm{i}}^{\prime} \nabla \mathrm{x}_{\mathbf{j}}^{\prime}
$$

Here, $x_{i}^{\prime}$ means $\xi$ or $\eta$ depending on the index $i$

$$
\mathbf{S}=\frac{1}{\operatorname{Re}} \operatorname{diag}(0,1,1)
$$

where Re is the Reynolds number. 
The Navier-Stokes equations are mixed parabolic/hyperbolic partial differential equations. According to the eigenvalue analysis of the hyperbolic part of the equations, the Jacobian matrices in the transformed coordinates have real eigenvalues

$$
\begin{aligned}
& \widetilde{\mathbf{A}}=\frac{\partial \widetilde{\mathbf{E}}}{\partial \widetilde{\mathbf{Q}}}=\mathbf{K}\left(\mathrm{U}, \xi_{\mathrm{x}}, \xi_{\mathrm{y}}\right) \\
& \left.\widetilde{\mathbf{B}}=\frac{\partial \widetilde{\mathbf{F}}}{\partial \widetilde{\mathbf{Q}}}=\mathbf{K}\left(\mathrm{V}, \eta_{\mathrm{x}}, \eta_{\mathrm{y}}\right)\right)
\end{aligned}
$$

where the matrix $\mathbf{K}$ is defined as

$$
\mathbf{K}\left(k, \mathrm{k}_{1}, \mathrm{k}_{2}\right)=\left[\begin{array}{ccc}
0 & \mathrm{k}_{1} & \mathrm{k}_{2} \\
\beta \mathrm{k}_{1} & \mathrm{k}+\mathrm{k}_{1} \mathrm{u} & \mathrm{k}_{2} \mathrm{u} \\
\beta \mathrm{k}_{2} & \mathrm{k}_{1} \mathrm{v} & \mathrm{k}+\mathrm{k}_{2} \mathrm{v}
\end{array}\right]
$$

Here, $\mathrm{k}_{1}$ and $\mathrm{k}_{2}$ are either $\xi_{\mathrm{x}}$ and $\xi_{\mathrm{y}}$ or $\eta_{\mathrm{x}}$ and $\eta_{\mathrm{y}}$ depending on the direction to be considered, and $\mathrm{k}=$ $k_{1} u+k_{2} v$. The eigenvalues of the matrix $K$ are given by

$$
\Lambda=\operatorname{diag}(k-c, k+c, k)
$$

where the equivalent speed of sound, $c$, is given as

$$
c=\sqrt{k^{2}+\beta\left(k_{1}^{2}+k_{2}^{2}\right)}
$$

Notice that one of the eigenvalues is negative. This means that the incompressible flow is equivalently "subsonic" in the sense of different signs of the eigenvalues and that $c$ will influence stiffness of the system. Thus, the direction of characteristics should be considered when applying boundary conditions. 


\section{Numerical Methods}

The residual vector including the fourth order artificial dissipation (Eq. 23) is defined as

$$
\hat{\mathbf{R}}=\frac{\partial \widetilde{\mathbf{E}}}{\partial \xi}+\frac{\partial \widetilde{\mathbf{F}}}{\partial \eta}-D^{2}(\mathrm{~J} \tilde{\mathbf{Q}})+\mathrm{D}(\mathrm{J} \widetilde{\mathbf{Q}})
$$

After spatial derivative terms were discretized, the governing equations were integrated either by the explicit Runge-Kutta time-stepping algorithm (Eq. 25) or by an Euler implicit method with approximate factorization (Beam and Warming, ref. 24). To reduce the computational effort, the artificial dissipation and the viscous part of the residual are calculated only once every global time level and kept unchanged during the four stages of the Runge-Kutta scheme. This does not deteriorate the stability of the time stepping algorithm.

The Euler implicit scheme with factorization for the incompressible Navier-Stokes equations results in

$$
\begin{aligned}
& {\left[\mathbf{I}+\Delta \mathrm{t}\left\{\frac{\partial}{\partial \xi} \widetilde{\mathbf{A}} \cdot-\frac{\partial}{\partial \xi}\left(\frac{S g_{11}}{\mathrm{~J}} \frac{\partial}{\partial \xi} \mathrm{J} \cdot\right)\right\}\right]} \\
& \cdot\left[\mathbf{I}+\Delta \mathrm{t}\left\{\frac{\partial}{\partial \eta} \widetilde{\mathbf{B}} \cdot-\frac{\partial}{\partial \eta}\left(\frac{S_{22}}{\mathrm{~J}} \frac{\partial}{\partial \eta} \mathrm{J} \cdot\right)\right\}\right] \Delta \widetilde{\mathbf{Q}}=-\Delta \mathrm{t} \hat{\mathbf{R}}
\end{aligned}
$$

\section{Time Step Limitations and Boundary Conditions}

The allowable time increments of the explicit scheme are severely restricted by the stability limit, while for an implicit scheme the time step restrictions are caused by the factorization errors. The time step is determined by considering the hyperbolic part of the system and the parabolic part of the system separately and by combining these time steps as suggested by MacCormack and Baldwin (ref. 19). The system becomes hyperbolic when viscosity is neglected. Then, the stability bound of the resulting system is determined by the CFL (Courant-Friedrichs-Lewy) number. The maximum allowable time steps for each of the coordinate directions are defined as

$$
\Delta t_{h \xi}=\frac{C F L}{|U|+c_{\xi}} \quad \Delta t_{h \eta}=\frac{C F L}{|V|+c_{\eta}}
$$

so that the combined maximum time step for the hyperbolic part of the system is defined by 


$$
\Delta t_{h}=\frac{\Delta t_{h} \xi t_{h \eta}}{\Delta t_{h \xi}+\Delta t_{h \eta}}
$$

When the convective part of the acceleration is neglected, the system becomes of parabolic type. The stability of the parabolic type system is dictated by the non-dimensional number $\sigma$ (von Neumann number). For each generalized coordinate direction, the maximum time steps are defined by

$$
\Delta \mathrm{t}_{\mathrm{p} \xi}=\frac{\sigma \mathrm{Re}}{\mathrm{g}_{11}} \quad \Delta \mathrm{t}_{\mathrm{p \eta}}=\frac{\sigma \mathrm{Re}}{\mathrm{g}_{22}}
$$

and the combined maximum time step for the parabolic part is given by

$$
\Delta \mathrm{t}_{\mathrm{p}}=\frac{\Delta \mathrm{t}_{\mathrm{p} \xi} \Delta \mathrm{t}_{\mathrm{p \eta}}}{\Delta \mathrm{t}_{\mathrm{p} \xi}+\Delta \mathrm{t}_{\mathrm{p} \eta}}
$$

The total maximum time step is estimated conservatively as

$$
\Delta t=\frac{\Delta t_{h} \Delta t_{p}}{\Delta t_{h}+\Delta t_{p}}
$$

For the explicit Runge-Kutta method, Eq. 46 was used to estimate the maximum time step. However, for the Euler implicit method, only CFL limitation was used to compute the time step, that is

$$
\Delta t=\frac{\Delta t_{h \xi} \Delta t_{h \eta}}{\Delta t_{h \xi}+\Delta t_{h \eta}}
$$

It was assumed that the flow is inviscid at the inlet and exit planes causing the system of equations to become hyperbolic in time near the inlet and exit. As stated earlier, the incompressible Navier-Stokes equations have one negative eigenvalue, and the rest of the eigenvalues are positive. Thus, one equation should be considered with two boundary conditions at the inlet. At the exit, two equations with one boundary condition must be applied. At the inlet, $u$ and v velocity vector components were specified, while the back pressure $\mathrm{p}$ was specified at the exit. The flow was assumed to be locally onedimensional at the inlet and exit boundaries in order to transform locally the equation into the characteristic form. At the solid wall, the velocity vector components, $u$ and $v$, were set to zero, and the surface pressure was extrapolated from the grid points next to the wall from the condition that $\frac{\partial p}{\partial n}=$ 0. 


\section{Residual Smoothing}

One of the successful attempts to accelerate the convergence of the Runge-Kutta scheme is Implicit Residual Smoothing (IRS) introduced by Jameson and Baker (ref. 25). With this method, it is possible to use much higher values of CFL. The residual is smoothed through the following equation

$$
\left[1-\theta \delta_{\xi}^{2}\right]\left[1-\theta \delta_{\eta}^{2}\right] \hat{\mathbf{R}}^{*}=\hat{\mathbf{R}}
$$

where $\delta^{2}$ designates the central difference operator for a respective second derivative, and $\theta$ is the smoothing coefficient. Thus, when using the IRS we have to solve two scalar tri-diagonal matrices. Since their coefficients are constants, the tri-diagonal matrices are decomposed into upper and lower bidiagonal matrices so that at every application of the IRS only forward and backward substitutions are needed to get the smoothed residual.

The application of the DMR method to incompressible Navier-Stokes equations differs from the formulation for its application to the Euler equations of gasdynamics only by the following term

$$
\mathbf{a}_{\mathrm{q}}^{\mathrm{m}}=\left[\frac{\partial}{\partial \xi} \widetilde{\mathbf{A}}^{\mathrm{t}} \cdot+\frac{\partial}{\partial \eta} \widetilde{\mathbf{B}}^{\mathrm{t}} \cdot-D^{2} \mathbf{J} \cdot+\mathrm{DJ} \cdot\right] \frac{\partial \Theta^{\mathrm{m}}}{\partial \omega_{\mathrm{q}}^{\mathrm{m}}}
$$

\section{Computational Results for Navier-Stokes Equations}

A steady, laminar, viscous flow normal to a solid wall (Hiemenz flow) was the first test case. Reason for this choice of the test case is that the analytic solution for the Hiemenz flow is known (Panton, ref. 26). The accuracy of the codes (the explicit Runge-Kutta method and the Euler implicit method) can be verified by comparing the computed solution with the analytic solution.

The flow corresponding to the Reynolds number 400 based on the free stream velocity and a body dimension, $\mathrm{R}_{0}$, of the wall was computed with and without the DMR method in conjunction with explicit and implicit codes. The computational grid consisted of $60 \times 29$ cells, and the dimensions of the computational domain were $\mathrm{H}=\mathrm{R}_{\mathrm{o}}$ and $\mathrm{L}=2 \mathrm{R}_{\mathrm{o}}$. In the case of an explicit Runge-Kutta (RK) method, the maximum allowable CFL number of 2.8 was used and the von Neumann number was $\sigma=0.4$. A small amount of the fourth order artificial dissipation was added to get a smooth solution $(\varepsilon=0.05)$. Using numerical experimentation it was found that the fastest convergence is obtained with the artificial 
compressibility coefficient $\beta=2$, and that the DMR method should be applied every 10 iterations by combining 3 consecutive solutions.

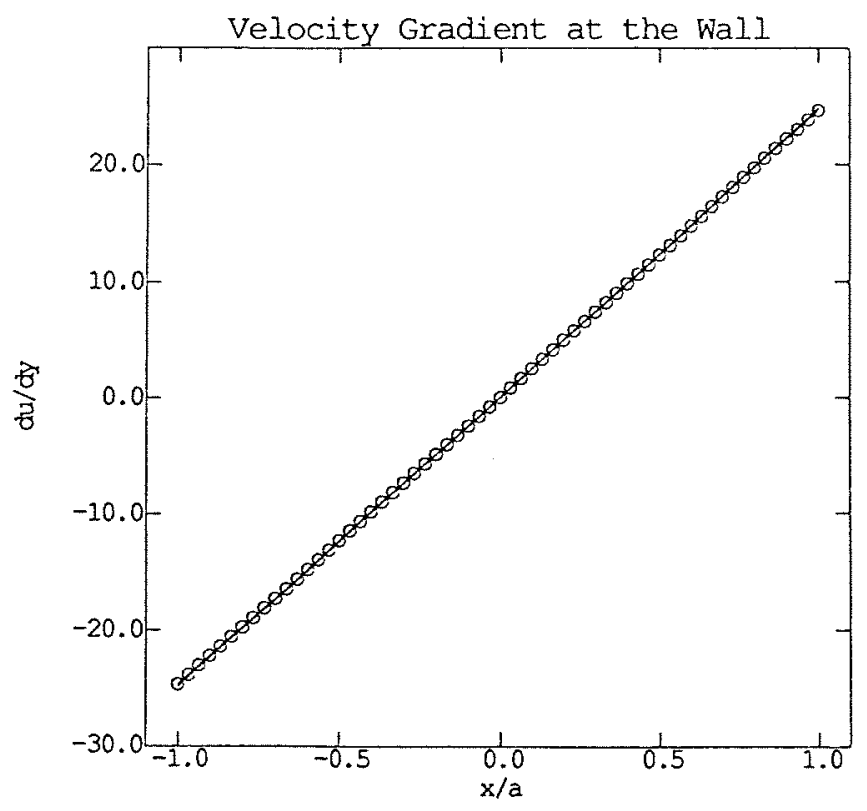

Figure 3 Distributions of wall surface velocity gradient for Hiemenz flow (RK: solid line; analytic solution: circles)
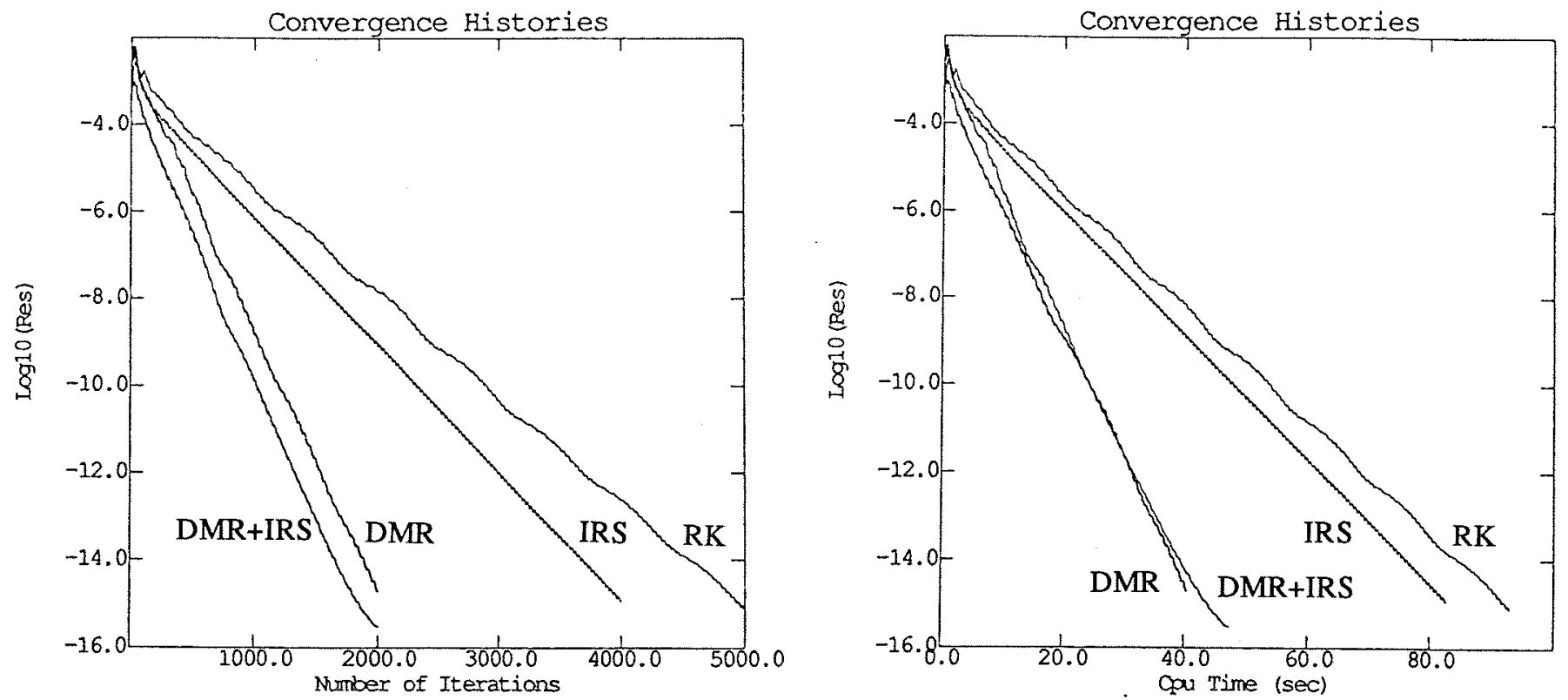

Figure 4 Convergence histories of the RK method for Hiemenz flow with Re $=400$

The computed distribution of the wall surface velocity gradient, $\frac{\partial u}{\partial y}$, was compared with that of the analytic solution (Fig. 3), showing an excellent agreement. Figure 4 shows that the residual was 
reduced 12 orders of magnitude in 5000 iterations without the DMR method, while the same reduction in residual could be achieved in 2000 iterations with the DMR method indicating $60 \%$ reduction in CPU time. The implicit residual smoothing was also implemented with and without the DMR method. The basic RK method gives the slowest convergence, the IRS gives faster convergence than the basic RK method, while the DMR method gave the second best convergence. The most rapid convergence in terms of the number of iterations was achieved by combining the implicit residual smoothing and the DMR method. However, the DMR method alone offered maximum time savings (over 55\%).

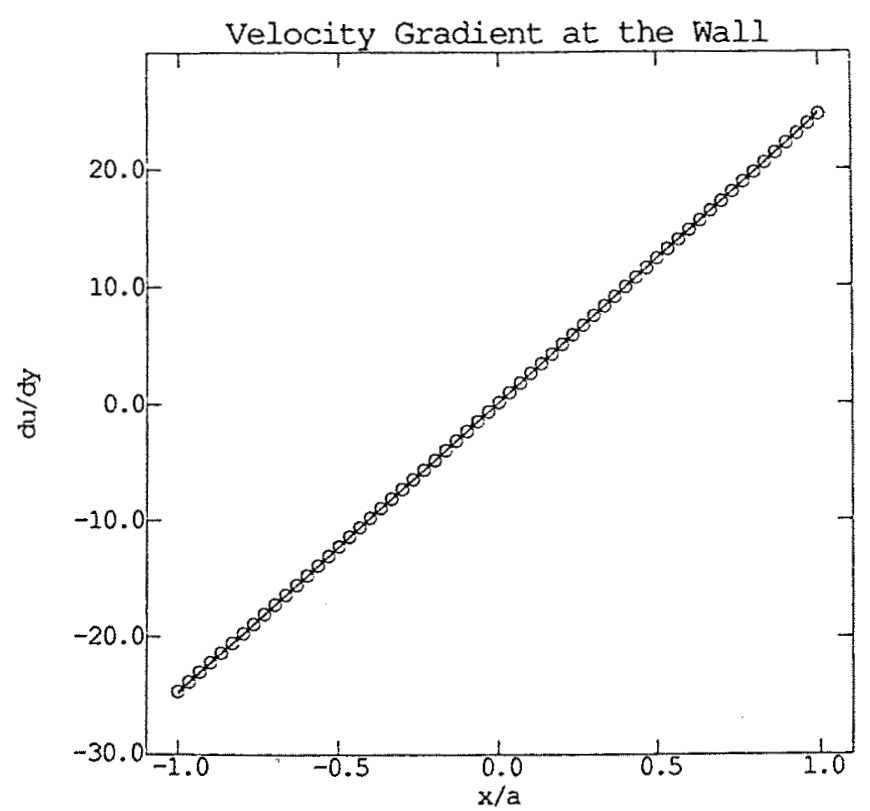

Figure 5 Distributions of wall surface velocity gradient for Hiemenz flow

(Euler implicit: solid line; analytic solution: circles)
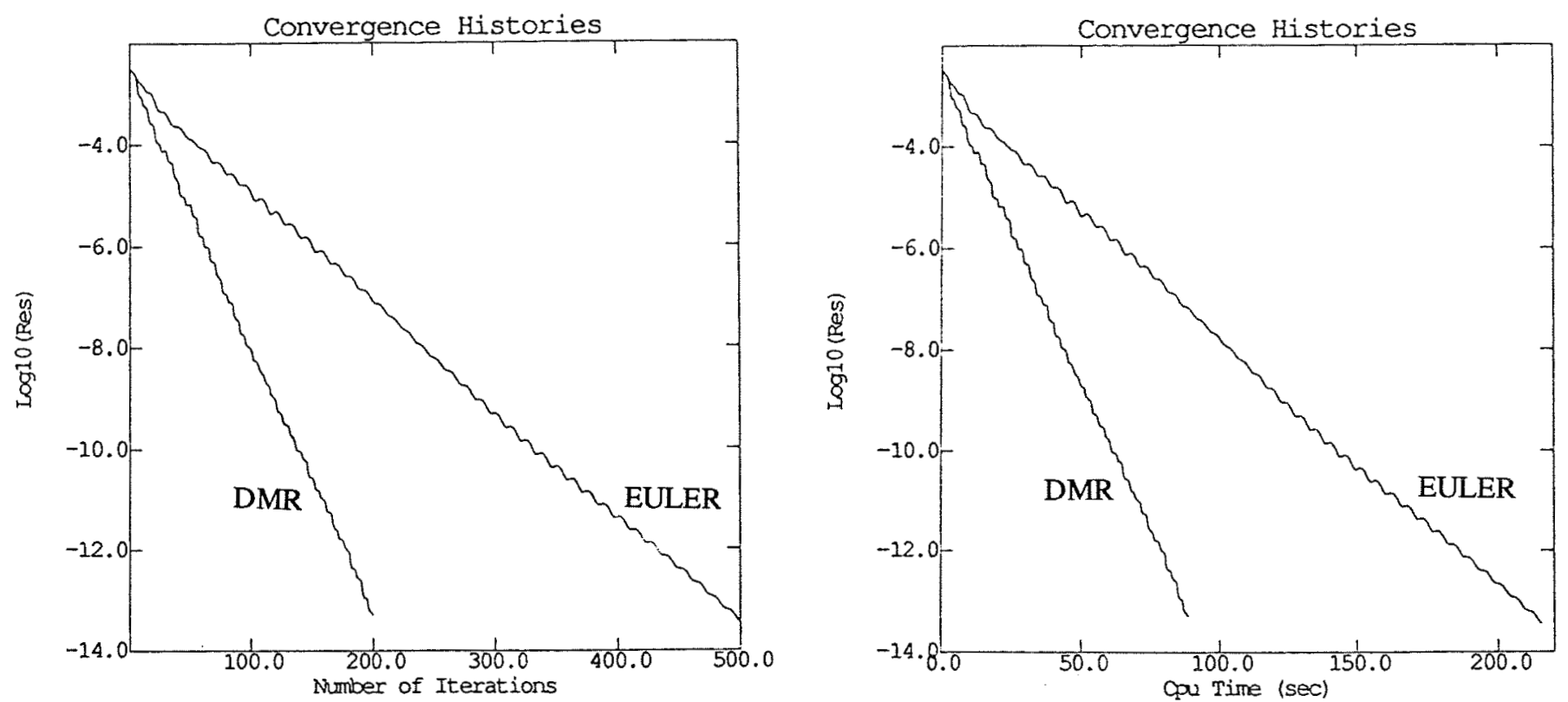

Figure 6 Convergence histories of the Euler implicit method for Hiemenz flow with $\mathrm{Re}=400$ 
The implicit code was also exercised for the test of the Hiemenz flow with the same conditions as in the test case for the explicit code $(\mathrm{Re}=400)$. The computed surface velocity gradient distribution was compared with the analytic solution (Fig. 5). Good agreement can be observed. CFL number of 10 was used in this computation. Also, the fourth order artificial dissipation with $\varepsilon=0.25$ was added. The optimal value of the artificial compressibility coefficient $\beta$ was found by numerical experiments to be $\beta=5$. The DMR method was found to give the fastest convergence when applied to the implicit Euler scheme every 5 iterations by combining 5 consecutive solutions. Figure 6 shows that the DMR method offers approximately $60 \%$ reduction in CPU time indicating that the DMR method can be successfully applied to implicit methods.
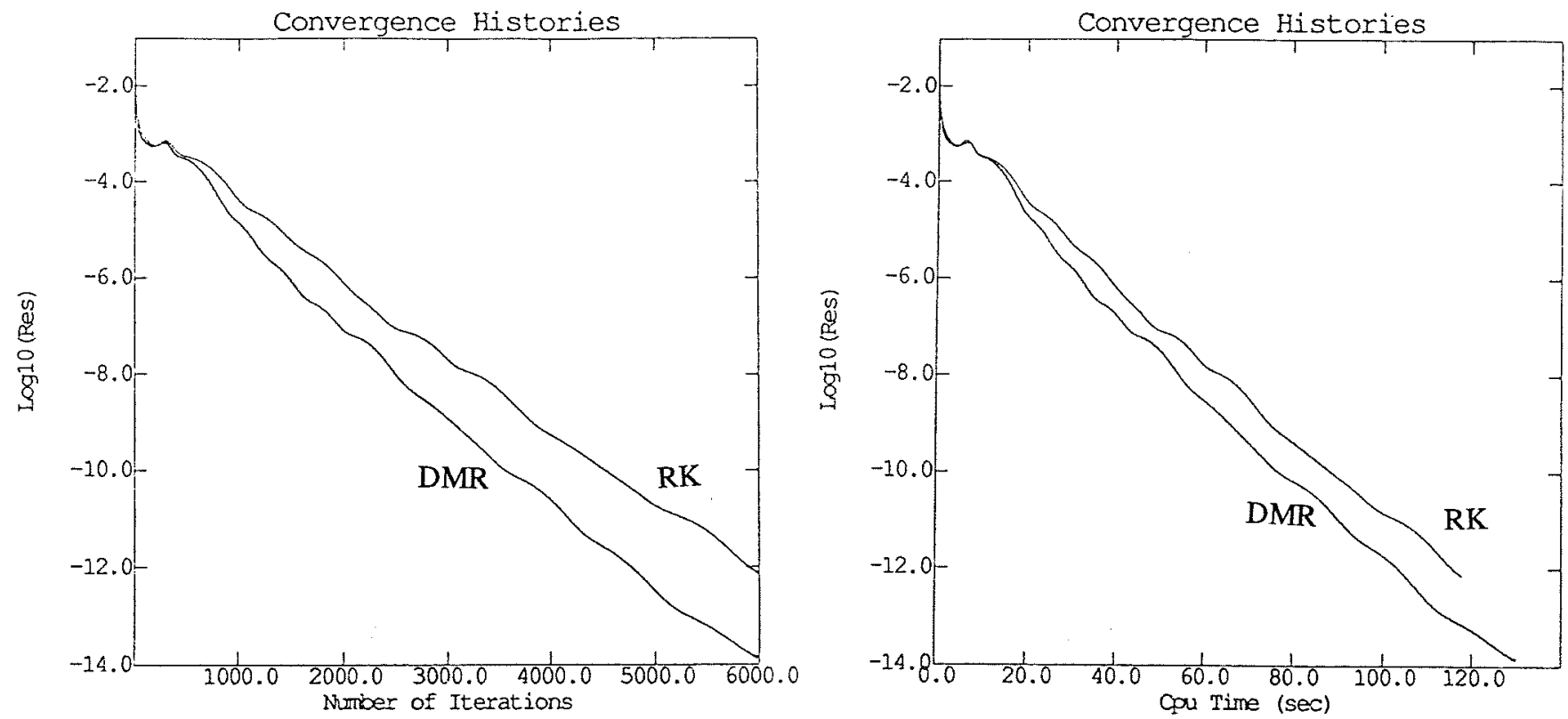

Figure 7 Convergence histories for viscous flow around a circular cylinder with $\operatorname{Re}=20(\mathrm{RK})$

The last test case was a laminar flow around a circular cylinder. The highly clustered grid of $66 \times 44$ cells was used. Flow with Reynolds numbers of 20 was computed with the RK method and the Euler implicit method. The CFL and von Neumann numbers were CFL $=2.8$ and $\sigma=0.4$, respectively, for the RK method, and CFL = 10 was used for the Euler implicit method. The DMR method was applied every 30 iterations for the RK method, while every 10 iterations for the Euler implicit method. For both methods, two consecutive solutions were used with the DMR method, though these combinations of the number of solutions and the frequency of the DMR application are not optimal. The artificial compressibility coefficient was $\beta=1$ for both methods. The convergence histories of the RK method and the Euler implicit method with and without the DMR method are presented in Figures 7 and 8 . The DMR method offers more savings with the Euler implicit method (30\%) than with the RK method (10\%). The wall pressure distributions and the wall vorticity distributions were compared with the computational results of Choi (1989) in Figures 9 and 10 showing reasonable agreement. 

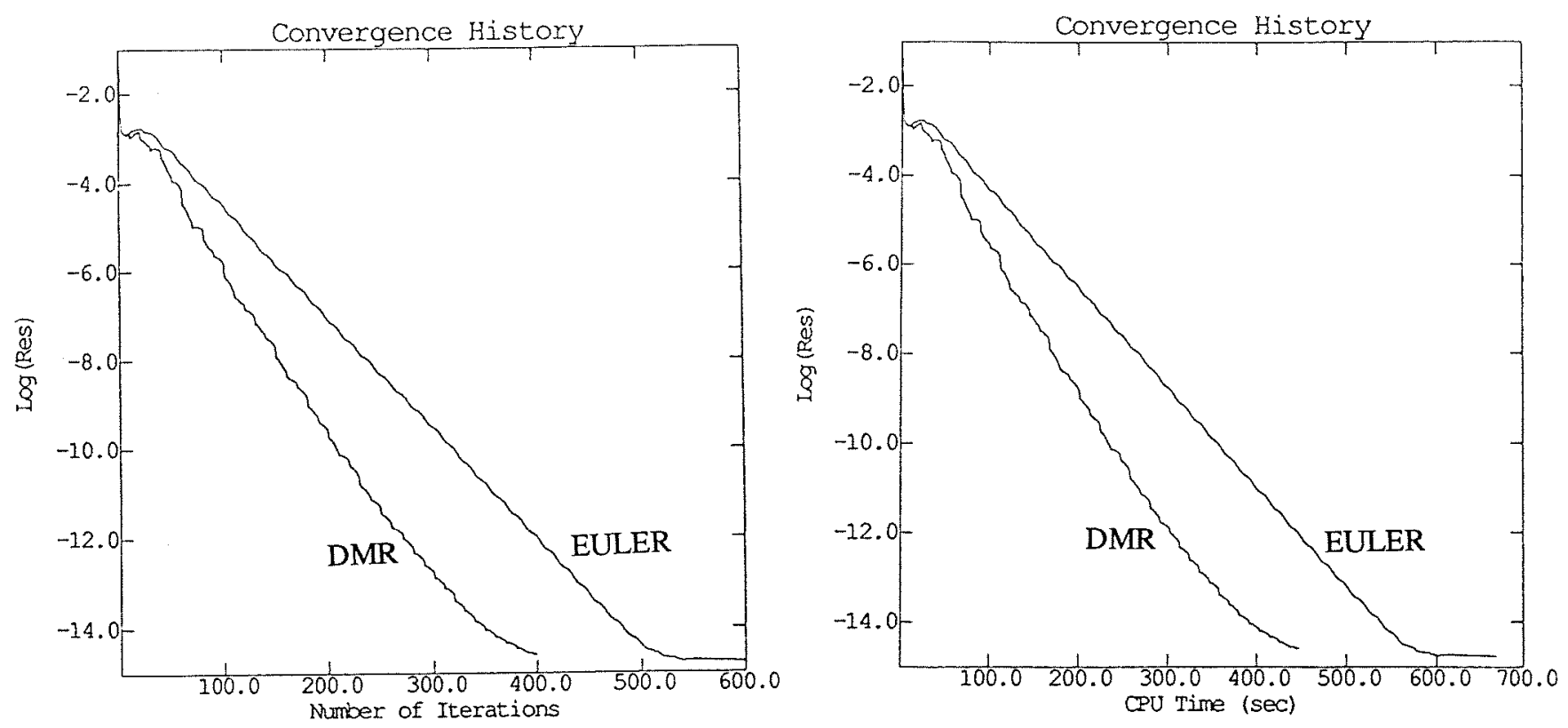

Figure 8 Convergence histories for viscous flow around a circular cylinder with $\operatorname{Re}=20$ (Euler implicit)

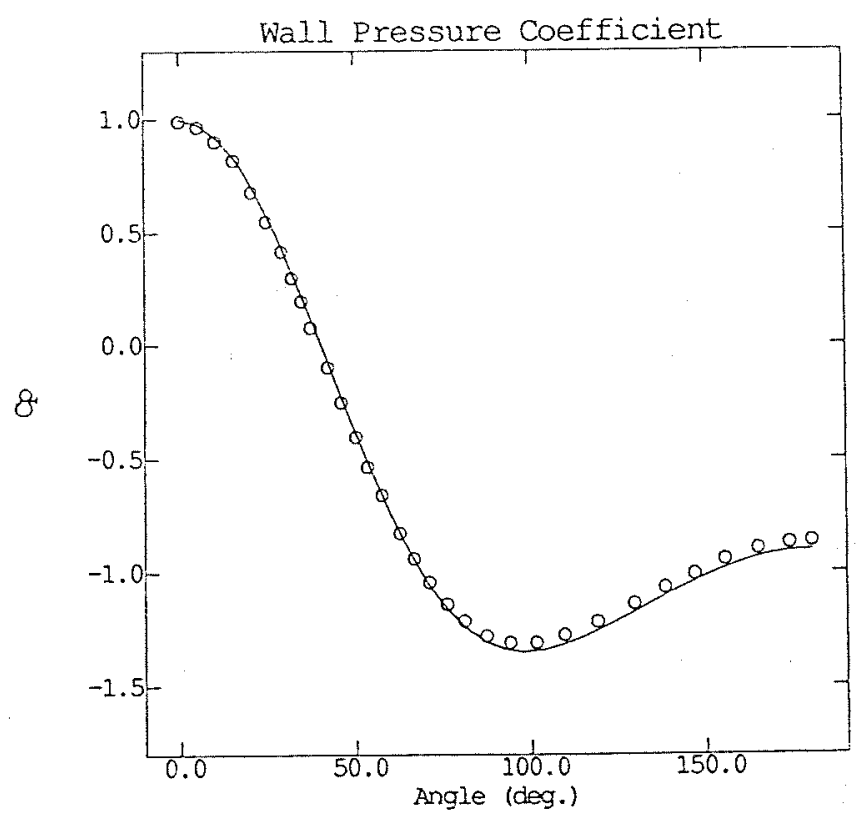

(a) Pressure coefficient

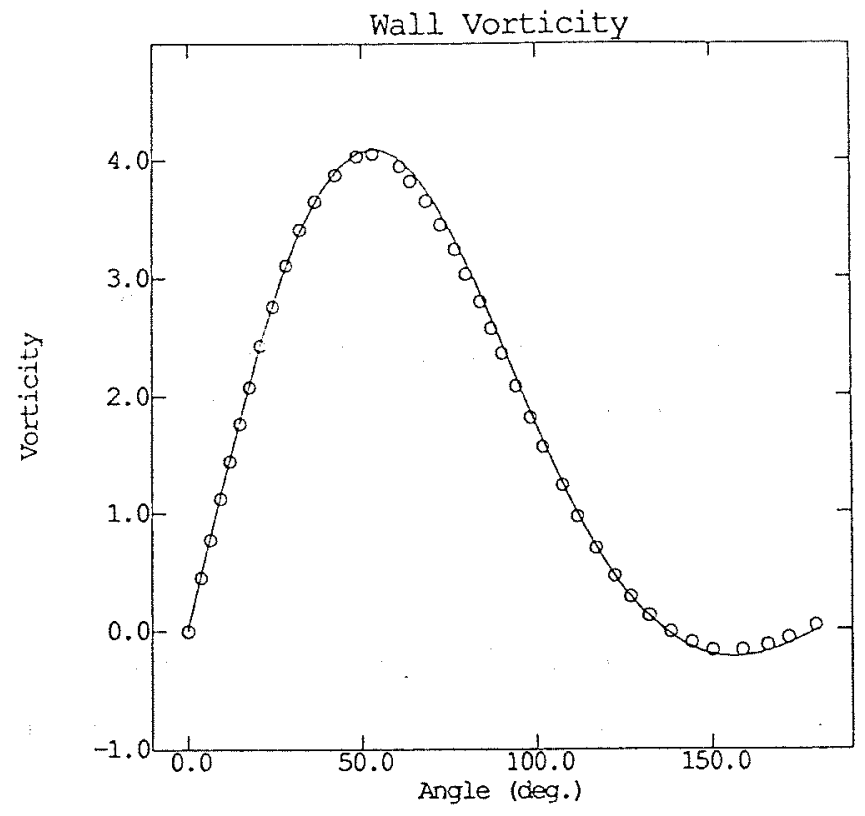

(b) Vorticity

Figure 9 Wall pressure coefficient distributions and vorticity distributions for flow around a circular cylinder at $\mathrm{Re}=20(\mathrm{RK})$ 


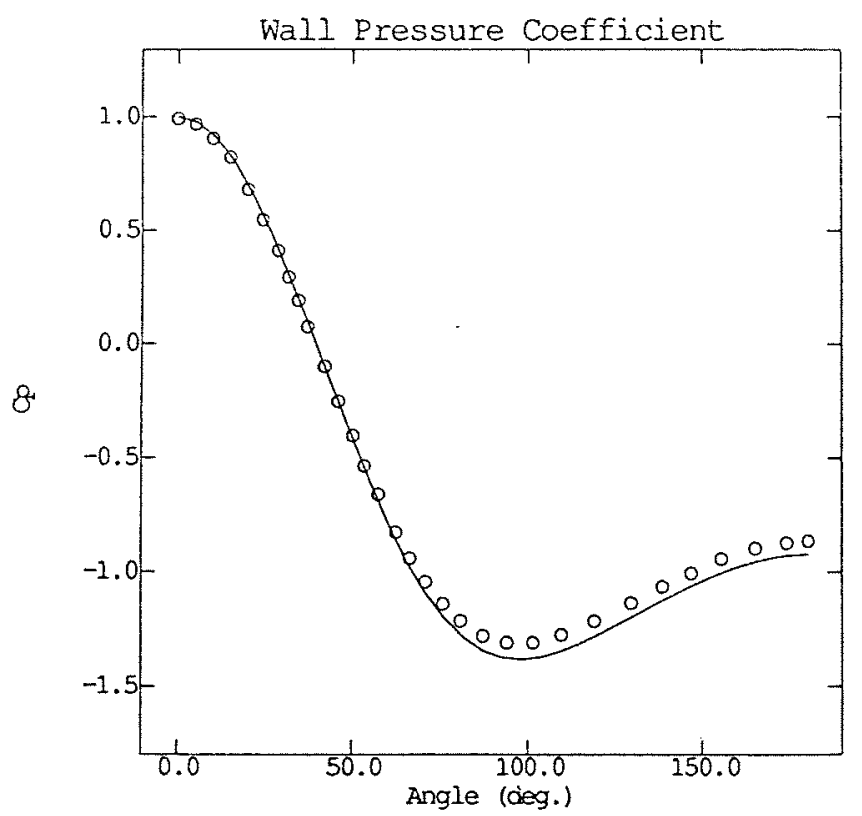

(a) Pressure coefficient

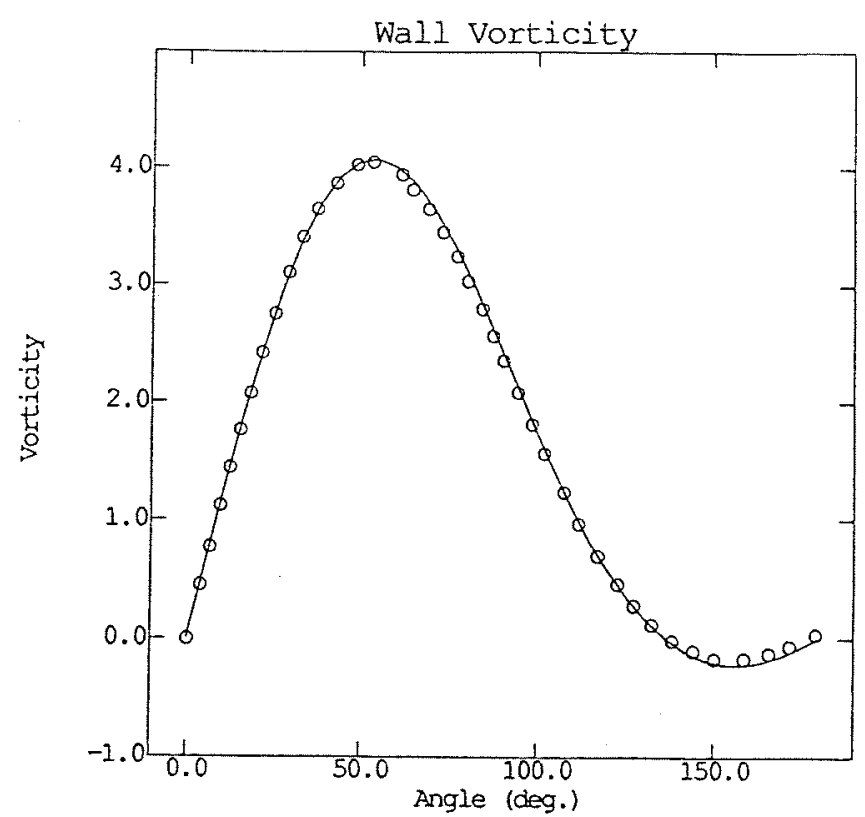

(b) Vorticity

Figure 10 Wall pressure coefficient distributions and vorticity distributions for flow around a circular cylinder at $\mathrm{Re}=40$ (Euler implicit)

\section{CONCLUSIONS}

The DMR method was found capable of reducing the computation time by $20-80 \%$ depending on the test case. When directly compared with an implicit residual smoothing, the DMR method performed consistently better and more reliably.

\section{REFERENCES}

1 Huynh, Q. Q, "Iterative Preconditioned Gradient-Newton Type methods for Modern Shock Computations," Dept. of Computer Sciences, Uppsala University, Report No. 107, 1987.

2 Faddeev, D. K. and Faddeeva, V. N, Computational Methods of Linear Algebra, Trans. by Williams, R. C., 1963, W. H. Freeman and Company. San Francisco, California.

3 Vinsome, P. K. W., "ORTHMIN, and Iterative Method for Solving Sparse Sets of Simultaneous Linear Equations," Proc. Fourth Symposium on Reservoir Simulation, Society of Petroleum Engineers of AIME, 1976. 
4 Young, D. M. and Jea, K. C., "Generalized Conjugate-Gradient Acceleration of Nonsymmetrizable Iterative Methods," Linear Algebra Appl., Vol. 34, 1980.

5 Saad, Y, and Schultz, M, "Conjugate Gradient-Like Algorithms for Solving Non-symmetric Linear Systems," Mathematics of Computation, Vol. 44, No. 170, 1985.

6 Wigton, L. B., Yu, N. J. and Young, D. P., "GMRES Acceleration of Computational Fluid Dynamics Codes," AIAA Paper 85-1494, 1985.

7 Hafez, M., Parlette, E., and Salas, M. D., "Convergence Acceleration of Iterative Solutions of Euler Equations for Transonic Flow Computations," AIAA Paper 85-1641, July 1985.

8 Huang, C. Y. and Dulikravich, G. S., "Fast Iterative Algorithms Based on Optimized Explicit Time-Stepping," Computer Methods in Applied Mechanics and Engineering, 63, Aug. 1987, pp. 15-36.

9 Lee, S., Dulikravich, G. S. and Dorney, D., "Distributed Minimal Residual (DMR) Method for Explicit Algorithms Applied to Nonlinear Systems," presented at the Conference on Iterative Methods for Large Linear Systems, Austin, Texas, Oct. 19-21, 1988.

10 Dulikravich, G. S., Dorney, D. J. and Lee, S., "Iterative Acceleration and Physically Based Dissipation for Euler Equations of Gasdynamics," Proceedings of ASME WAM'88, Symposia on Advances and Applications in Computational Fluid Dynamics, edited by O. Baysal, FED - Vol. 66, Chicago, IL, Nov. 28 - Dec. 2, 1988, pp. 81-92.

11 Lee, S., Dulikravich, G. S. and Dorney, D. J., "Acceleration of Iterative Algorithms for Euler Equations of Gasdynamics," AIAA Paper 89-0097, Reno, NV, Jan. 1989; Also, accepted for publication in AIAA Journal, 1990.

12 Lee, S. and Dulikravich, G. S., "Accelerated Computation of Viscous, Steady Incompressible Flows," ASME paper 89-GT-45, Gas Turbine and Aeroengine Congress and Exposition, Toronto, Canada, June 4-8, 1989.

13 Lee, S. and Dulikravich, G. S., "A Fast Iterative Algorithm for Incompressible Navier-Stokes Equations," Proceedings the 10th Brazilian Congress of Mechanical Engineering, Rio de Janeiro, Brazil, Dec. 7-10, 1989.

14 Lee, S., Acceleration of Iterative Algorithms for Euler and Navier-Stokes Equations, $\mathrm{Ph} . \mathrm{D}$. Thesis, Department of Aerospace Engineering, Pennsylvania State University, May 1990.

15 Briley, W. R, McDonald, H. and Shamroth, S. J., "A Low Mach Number Euler Formulation and Application to Time-Iterative LBI Schemes," AIAA Journal, Vol. 21, No. 10, Oct. 1983.

16 Choi, Y., Computation of Low Mach Number Compressible Flow, Ph. D. Thesis, Department of Mechanical Engineering, Pennsylvania State University, 1989.

17 Turkel, E, "Preconditioned Methods for Solving the Incompressible and Low Speed Compressible Equations," Journal of Computational Physics, Vol. 72, No. 2, Oct. 1987, pp. 277-298. 
18 Jameson, A., Schmidt, W., and Turkel, E., "Numerical Solutions of the Euler Equations by Finite Volume Methods Using Runge-Kutta Time-Stepping Scheme," AIAA paper 81-1259, Palo Alto, CA, June, 1981.

18 Steger, J. L. and Kutler, P., "Implicit Finite-Difference Procedure for the Computation of Vortex Wakes," AIAA Journal, Vol. 15, No. 7, July 1977, pp. 581-590.

19 MacCormack, R. W. and Baldwin, B. S., "A Numerical Method for Solving the Navier-Stokes Equations With Application to Shock-Boundary Layer Interaction," AIAA Paper 75-1, Jan. 1975.

20 Chakravarthy, S. R., "Euler Equations - Implicit Schemes and Implicit Boundary Conditions," AIAA paper 82-0228, AIAA 20th Aerospace Sciences Meeting, Orlando, Florida, Jan. 11-14, 1982.

21 Chorin, A. J., "A Numerical Method for Solving Incompressible Viscous Flow Problems," Journal of Computational Physics, Vol. 2, 1967, pp. 12-26.

22 Choi, D. and Merkle, C. L., "Application of Time-Iterative Schemes to Incompressible Flows," AIAA Journal, Vol. 23, No.10, Oct. 1985, pp. 1518-1524.

23 Kwak, D., Chang, J. L. C., Shanks, S. P. and Chakravarthy, S. R., "An Incompressible NavierStokes Flow Solver in Three Dimensional Curvilinear Coordinate System Using Primitive Variables," AIAA Journal, Vol. 24, No. 3, March 1986, pp. 390-396.

24 Beam, R. M. and Warming, R. F., "Implicit Numerical Methods for the Compressible NavierStokes and Euler Equations," Lecture Notes Series 1982-04, von Karman Institute for Fluid Dynamics, Belgium, 1982.

25 Jameson, A., and Baker, T. J., "Solution of the Euler Equations for Complex Configurations," Proceedings of AIAA 6th Computational Fluid Dynamics Conference, AIAA, New York, 1983, pp. 293-302.

26 Panton, R. L., Incompressible Flow, John Wiley and Sons, Inc., New York, New York, 1984. 